\title{
Diagnosis and Management of Fat Overload Syndrome in an Elderly Man
}

\author{
Zhanqiang Zhang, Honglan Yu, Kaitao Yuan, Hanping Shi* \\ Department of Surgery, The First Affiliated Hospital, Sun Yet-sen University, Guangzhou, China \\ Email: ${ }^{*} z z q-006 @ 163 . c o m$
}

Received 10 August 2014; revised 9 September 2014; accepted 8 October 2014

Copyright (C) 2014 by authors and Scientific Research Publishing Inc.

This work is licensed under the Creative Commons Attribution International License (CC BY). http://creativecommons.org/licenses/by/4.0/

cc) (7)

\begin{abstract}
Fat overload syndrome is a rare complication of intravenous fat emulsion therapy. It is due to infusion rate and/or dose of triglycerides lipid emulsion surpassing fat clearance capacity of the body. Several reports in the literature described fat overload syndrome which was caused by rapid infusion of lipid emulsions, all with soybean-based lipid emulsions. We describe one patient who received parenteral nutrition with $20 \%$ medium-chain and long-chain triglycerides lipid perioperatively for volvulus of sigmoid colon, followed by a precipitous drop in blood platelet suggestive of fat overload syndrome. After a complete withdrawal of medium-chain and long-chain triglycerides lipid emulsion, the platelet levels gradually returned to be normal.
\end{abstract}

\section{Keywords}

Fat Overload Syndrome, Parenteral Nutrition, Thrombocytopenia

\section{Introduction}

Fat emulsions are an important component of parenteral nutrition (PN) as a source of energy substrate and essential fatty acids. Fat overload syndrome, caused by overload triglycerides lipid emulsion surpassing fat clearance capacity of the body, is a rare complication of parenteral nutrition. It is characterized by headaches, fever, jaundice, hepatosplenomegaly, respiratory distress, and spontaneous hemorrhage. We present a 98-year-old patient who appeared scattered petechiae and thrombocytopenia after the operation for volvulus of sigmoid colon. The final diagnosis of fat overload syndrome was made after we exclused other condition causing thrombocytopenia.

*Corresponding author.

How to cite this paper: Zhang, Z.Q., Yu, H.L., Yuan, K.T. and Shi, H.P. (2014) Diagnosis and Management of Fat Overload Syndrome in an Elderly Man. Case Reports in Clinical Medicine, 3, 554-556. http://dx.doi.org/10.4236/crcm.2014.310120 


\section{Case Report}

A 98-year-old man presented with a 7-day history of abdominal pain and abdominal distention. His past medical history was significant forcerbral infarction. On examination, the abdomen was distended with no mass or peritonitis. The bowel sounds were hypoactive with occasional high pitched rushes. No hernias were identified. A rectal examination revealed no masses and no stool in the rectal vault. His laboratory work up showed WBC $12.05 \times 10^{9} / \mathrm{L}$, RBC $3.8 \times 10^{12} / \mathrm{L}$, HCT 34.3\%, PLT $359 \times 10^{9} / \mathrm{L}$, BUN 10.7 mmol/L, creatinine 165.4 umol/L. The Liver function and blood clotting function were normal. The abdominal radiographs and CT scan showed a dilated bowel with air-fluid levels, and suspected volvulus of sigmoid colon. Exploratory laparotomy and the sigmoid colon reposition and fixation was performed. None of these procedures was associated with abnormal or excessive blood loss. The process of diagnosis and treatment for thrombocytopenia was as follows:

On the 13th day after surgery, there was the onset of scattered petechiae and thrombocytopenia

$\downarrow$ Methylprednisolone $480 \mathrm{mg}$, gamma globulin $20 \mathrm{~g}$, and an infusion of platelets $2 \mathrm{U}$

On the 19th day after surgery, a reexamination revealed a progressive decrease of platelets again

$\downarrow$ Reinfusion of platelets $1 \mathrm{U}$

On the 21st day after surgery, a reexamination of items of rheumatism and thyroid function as well as indicators of erythematosus were all negative, without positive findings from bone marrow puncture

$\downarrow$

Exclusion of secondary thrombocytopenia caused by autoimmune diseases

$\downarrow$ Examinations of EDTA, anticoagulant tube and heparin anticoagulant tube, and a routine test of blood were separately taken

Exclusion of false thrombocytopenia

$\downarrow$ A combination with antibiotics and after controlling pulmonary infectionthere was a recurrence of thrombocytopenia

Exclusion of thrombocytopenia caused by severe infections

$\downarrow$ After a complete withdrawal of antibiotics and other drugs, there still was a recurrence of thrombocytopenia Exclusion of thrombocytopenia caused by other drugs

$\downarrow$ After a complete withdrawal of medium-chain and long-chain triglycerides lipid emulsion, platelet levels gradually returned to be preoperative ones

Considering thrombocytopenia caused by long-term infusion of medium-chain and long-chain triglycerides lipid emulsion, the condition was identified to be fat overload syndrome after discussion.

\section{Discussion}

As early as 1976, American pediatric surgeons, Robert et al. [1], reported a case of fat overload syndrome caused by soybean oil emulsion. In this case, on the 114th day of infusion into the sick child, there was the onset of a series of clinical manifestations including hepatosplenomegaly, oliguria, Cheyne-Stokes respiration, gallop rhythm, spontaneous mucocutaneous hemorrhage, and thrombocytopenia. On the 5th day when discontinued the infusion of triglycerides lipid emulsion, it was basically cleared in the sick child. Since the 6th day, abnormal clinical manifestations as well as results of laboratory tests had gradually returned to be normal.

To the best of our knowledge, Fat overload syndrome is due to infusion rate and/or dose of triglycerides lipid emulsion surpassing fat clearance capacity of the body, characterized by an elevated triglyceride. Several reports in the literature describe fat overload syndrome caused by soybean-based lipid emulsions [2] [3]. However, recently published case series showed that rapid infusion of a fish oil-based emulsion was well tolerated with no symptoms of fat overload syndrome [4]. It is characterized by headaches, fever, jaundice, hepatosplenomegaly, respiratory distress, and spontaneous hemorrhage [5]. Other symptoms include anemia, leukopenia, thrombocytopenia, low fibrinogen levels, and coagulopathy [6]. Clinically, it is common in children, the elderly, and patients with end-stage diseases as well as lipodystrophy caused by any other reasons. It was a 98 years old patient in this case. Although blood lipid level was normal on admission, there might be a fat clearance disturbance.

The main reasons for fat overload syndrome could be attributed to two aspects: 1) The fat clearance capacity of this patient was normal but overdose of triglycerides lipid emulsion was infused; 2) The dosage of triglycerides lipid emulsion was normal but the fat clearance capacity of the body reduced. In this case, the pathogenesis of thrombocytopenia was involved in many aspects: 1) Under the effect of cyclooxygenase and lipoxygenase, n-3 PUFA generated PAF, leading to a decrease in the number of available platelets; 2) n-3 PUFA, embedded 
into the phospholipid bilayer, changed the original membrane structure and membrane fluidity and hence resulted in a decrease in the number of lipid rafts, membrane signaling protein translocation, and etc., leading to a delayed immune response; 3) The phagocytosis of platelets to excess lipids incurred platelet dysfunction and it would also affect the coagulation function.

Once there was the onset of above symptoms and suspected to be fat overload syndrome, the infusion of triglycerides lipid emulsion or parenteral nutrient solution, containing triglycerides lipid emulsion, should be discontinued immediately. Meanwhile, blood fat should be monitored. In consideration of the condition, a symptomatic and supportive treatment should be given. If there was the onset of complications such as hemolysis, the infusion of red blood cells, albumin, frozen plasma, and etc. should be considered. Under the condition that no other treatment was effective, plasma exchange could be applied to clear excess blood fat in circulation [7].

\section{Conclusion}

Fat overload syndrome is a rare complication of intravenous fat emulsion therapy. In our case, diagnosis of fat overload syndrome is difficult; a positive diagnosis can be made only after ruling out other condition. Once there was the onset of above symptoms and suspected to be fat overload syndrome, stopping the infusion of triglycerides lipid emulsion immediately maybe the best treatment.

\section{References}

[1] Belin, R.P., Bivins, B.A., Jona, J.Z. and Young, V.L. (1976) Fat Overload with a 10\% Soybean Oil Emulsion. Archives of Surgery, 111, 1391-1393. http://dx.doi.org/10.1001/archsurg.1976.01360300081013

[2] Heyman, M.B., Storch, S. and Ament, M.E. (1981) The Fat Overload Syndrome. Report of a Case and Literature Review. American Journal of Diseases of Children, 135, 628-630. http://dx.doi.org/10.1001/archpedi.1981.02130310034012

[3] Campbell, A.N., Freedman, M.H., Pencharz, P.B. and Zlotkin, S.H. (1984) Bleeding Disorder from the "Fat Overload” Syndrome. Journal of Parenteral and Enteral Nutrition, 8, 447-449. http://dx.doi.org/10.1177/0148607184008004447

[4] Gura, K.M. and Puder, M. (2010) Rapid Infusion of Fish Oil-Based Emulsion in Infants Does Not Appear to Be Associated with Fat Overload Syndrome. Nutrition in Clinical Practice, 25, 399-402. http://dx.doi.org/10.1177/0884533610373770

[5] Goulet, O., Girot, R., Maier-Redelsperger, M., Bougle, D., Virelizier, J.L. and Ricour, C. (1986) Hematologic Disorders Following Prolonged Use of Intravenous Fat Emulsions in Children. Journal of Parenteral and Enteral Nutrition, 10, 284-288. http://dx.doi.org/10.1177/0148607186010003284

[6] Brans, Y.W., Dutton, E.B., Andrew, D.S., Menchaca, E.M. and West, D.L. (1986) Fat Emulsion Tolerance in Very Low Birth Weight Neonates: Effect on Diffusion of Oxygen in the Lungs and on Blood pH. Pediatrics, 78, 79-84.

[7] Kollef, M.H., McCormack, M.T., Caras, W.E., Reddy, V.V. and Bacon, D. (1990) The Fat Overload Syndrome: Successful Treatment with Plasma Exchange. Annals of Internal Medicine, 112, 545-546.

http://dx.doi.org/10.7326/0003-4819-112-7-545 
Scientific Research Publishing (SCIRP) is one of the largest Open Access journal publishers. It is currently publishing more than 200 open access, online, peer-reviewed journals covering a wide range of academic disciplines. SCIRP serves the worldwide academic communities and contributes to the progress and application of science with its publication.

Other selected journals from SCIRP are listed as below. Submit your manuscript to us via either submit@scirp.org or Online Submission Portal.
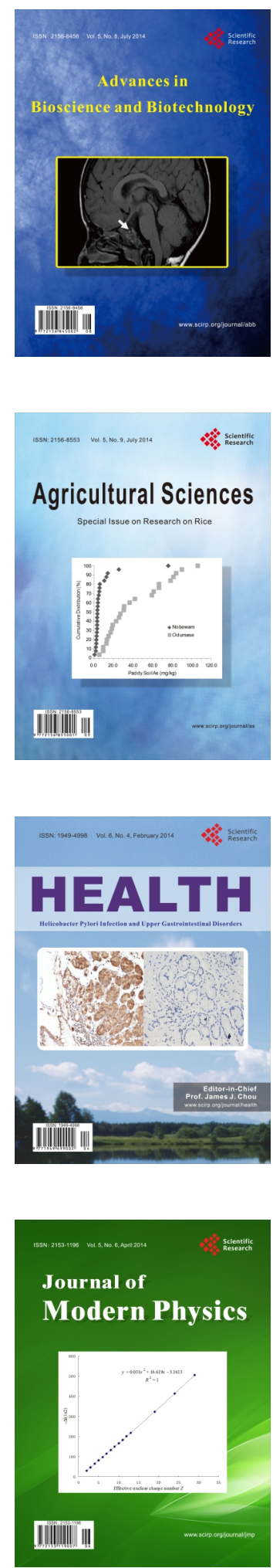
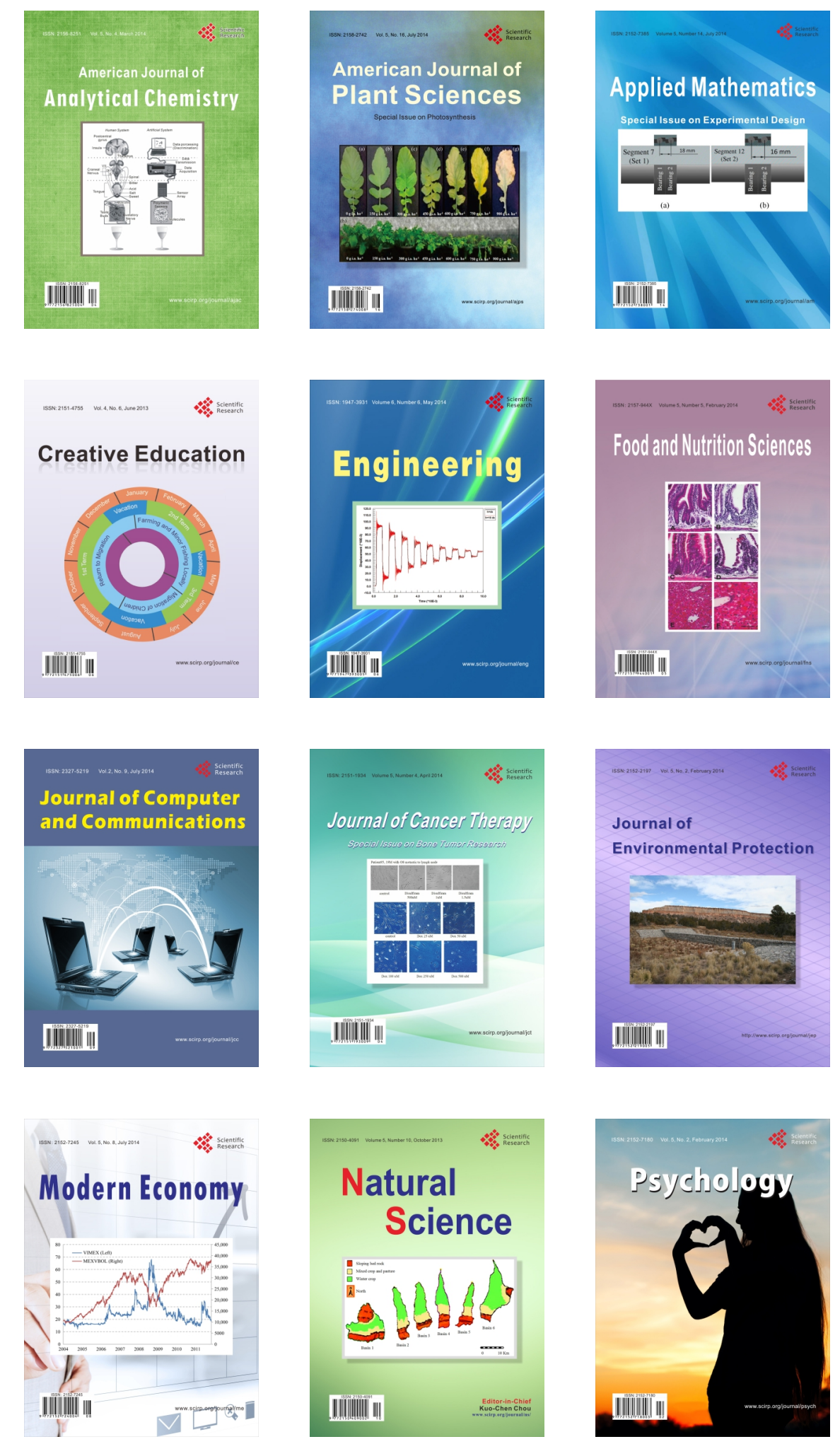